\title{
Functional Analysis of Phenotypic Behaviors of a 5-Year-Old Male with Novel 4q21 Microdeletion
}

\author{
Ashley Fee ${ }^{1} \cdot$ Nathan Noble $^{2} \cdot$ Maria G. Valdovinos ${ }^{1}$
}

Received: 11 June 2015 /Revised: 15 September 2015 / Accepted: 16 September 2015 / Published online: 7 October 2015

(C) American Academy of Pediatric Neuropsychology 2015

\begin{abstract}
The 4q21 microdeletion syndrome is a relatively newer syndrome that has been recently characterized by mild to severe intellectual disability, growth delay, and behavioral problems. The literature reports that aggression and self-injurious behavior have been identified as elements of the 4q21 microdeletion behavioral phenotype (Bonnet et al. in Journal of Medical Genetics, 47, 377-384, 2010; Strehle et al. in American Journal of Medical Genetics Part A, 158A, 2139-2151, 2012; Tsang et al. in American Journal of Medical Genetics Part A, 158A, 2606-2609, 2012). In this case report, functional assessment of aggression and self-injurious behavior of a 5-year-old male with 4q21 microdeletion syndrome was completed to determine the function of behavior and evaluate the effectiveness of behavioral intervention. The assessment result informed treatment subsequently produced a decrease in the occurrence of problem behaviors. This is one of the first functional assessments completed on behaviors associated with $4 \mathrm{q} 21$ microdeletion. Furthermore, this study exemplifies the utility of functional assessments in assessing and treating behaviors in those with known genetic conditions.
\end{abstract}

Keywords 4q21 microdeletion · Aggression · Self-injury · Functional assessment

Maria G. Valdovinos

maria.valdovinos@drake.edu

1 Department of Psychology, Drake University, 2507 University Avenue, Des Moines, IA 50311, USA

2 Blank Children's Developmental Clinic, Blank Children's Hospital, Des Moines, IA 503, USA
Recent advances in genetics allow for the evaluation of chromosomal disorders at specific loci on the chromosome. One such newly identified syndrome is 4q21 microdeletion syndrome (Bonnet et al. 2010). This syndrome involves the long arm of the fourth chromosome and can affect five known genes: PRKG2 (located on 4q13.1-q21.1), RASGEF1B (located on 4q21.21), HNRNPD (located on 4q21), HNRPDL (located on chromosome 6), and ENOPH1 (located on 4q21.22; Bonnet et al. 2010). The research regarding this specific microdeletion is sparse particularly with regard to the behavioral phenotype associated with the syndrome.

Thus far, the identified characteristics of 4q21 microdeletion include intellectual disability, potentiation in hippocampal neurons leading to specific deficits in learning and memory, severe growth restrictions, and the absence of speech (Bonnet et al. 2010; Strehle et al. 2012; Tsang et al. 2012). Mild to severe intellectual disability and severe developmental delays are also common in individuals with a $4 \mathrm{q} 21$ microdeletion. Facial dysmorphism and small hands and feet have often been reported in individuals with 4q21 microdeletion syndrome (DukesRimsky et al. 2010; Tsang et al. 2012). Finally, although another syndrome involving the fourth chromosome (4q deletion syndrome) presents with behavioral problems (i.e., aggression and self-injury; Strehle et al. 2012), the literature regarding 4 q21 microdeletion syndrome is extremely limited.

As with many microdeletion syndromes, clinical variability is often observed and is attributed to the specific genes impacted. For example, the HNRPDL gene has been associated with dominant forms of limb-girdle muscular dystrophies (LGMD) affecting the voluntary movement of hips and shoulders (Nigro and Savarese 2014). Furthermore, the loss of PRKG2 and RASGEF1B genes is associated with growth delay commensurate with the short stature often observed in individuals with 4q21 microdeletions (Bhoj et al. 2013; Gudbjartsson et al. 2008). The absence of PRKG2 and RASGEF1B genes is 
associated with the development of renal cysts (Tsang et al. 2012). Researchers have linked anxiety and depression (i.e., stress sensitivity) modulation to ENOPH1 gene expression in mice (Barth et al. 2014). Additionally, the RASGEF1B gene appears to account for the neurodevelopmental features in individuals with 4q21 microdeletion (Dukes-Rimsky et al. 2010).

Given that behavior problems, including aggression and self-injurious behaviors, have been associated with $4 \mathrm{q} 21$ microdeletion (Bonnet et al. 2010; Strehle et al. 2012; Tsang et al. 2012) further analyses of the behavioral characteristics are warranted to understand behavioral phenotype potentially associated with this relatively new syndrome. Functional analysis methodology developed by Iwata and colleagues (1994) has been heavily utilized to explore the function of problem behaviors in individuals with intellectual and developmental disabilities. Within functional analysis, variables which are thought to maintain or cause problem behavior, and mirror the child's everyday life, are systematically manipulated to determine which stimuli may be controlling behavior. In addition to manipulations of stimuli hypothesized to evoke and reinforce problem behavior, manipulations can also target adaptive behavior to confirm the hypothesized function of behavior. Furthermore, these methods have been proven both reliable and valid for the identification of behavior functions (Anderson et al. 1999).

Indeed, behavioral assessments such as these have been used to identify the function of behavior associated with biological conditions. For example, functional analysis methodology was used to determine the function of self-injurious behavior of an individual with Donnai-Barrow syndrome (Roane et al. 2012). What was unusual about this case was that the behaviors exhibited by the child had not been described in the existing literature. An effective treatment was designed and was informed by the results of the functional analysis conducted. Additional research has found that indirect assessments of behavior function (e.g., questionnaires) have also yielded information that can inform the behavioral phenotype of problem behaviors associated with genetic conditions (e.g., aggression and Smith-Magenis syndrome; Sloneem et al. 2011). Determining the function of behavior is important as it can lead to effective, individualized behavior treatment plans which aim to eliminate problem behaviors. The purpose of this case study was to use functional analysis methodology to determine the function of aggressive and self-injurious behaviors exhibited by a young male diagnosed with $4 \mathrm{q} 21$ microdeletion.

\section{Methods}

\section{Participant and Setting}

Julian (pseudonym) was a 5-year-old male with an 855 -kb de novo deletion of 4q21.22 affecting 12 genes. Additional copy number variants found in a microarray analysis included a confirmatory deletion of $6 \mathrm{q} 14.1$ found in the father, and a confirmatory maternally derived duplication of $21 \mathrm{q} 22.2$. Parental testing revealed the $6 \mathrm{q}$ deletion was paternally derived while the $21 \mathrm{q}$ duplication was maternally derived. The $4 \mathrm{q}$ deletion was de novo. Both of the patient's parents were typically developing and healthy. Julian was also diagnosed with intellectual disability disorder (moderate), language disorder, and hearing loss.

Julian's reported problem behaviors were aggression (pinching, grabbing, and hitting people and property), selfinjurious behavior (hitting his head on the table, making himself vomit or gag), dropping to the floor, and elopement (leaving the immediate area). At the time of the assessment, Julian was prescribed aripiprazole (Abilify) $2.5 \mathrm{mg}$ daily. His developmental pediatrician had referred Julian for assessment of problem behavior.

Assessment of Julian's behavior was conducted in an examination room in a children's hospital. This room was approximately $25 \times 45 \mathrm{ft}$ and contained an examining table, a writing table, four chairs, a couch, a counter that ran along one long wall of the room, and an anchored television. One of the short walls in the room contained darkened glass so the observers could see into the room (an observation room was adjacent to the examination room). The examination room was also equipped with a camera for easier viewing and hearing from the observation room.

\section{Procedure}

Prior to Julian's appointment, a Functional Assessment Interview (O’Neill et al. 1997) was conducted via telephone to gather additional information to assess the patient's history of problem behavior, conditions under which the behaviors were most likely to occur, and to identify any potential stimuli that function to strengthen or weaken the behavior (i.e., reinforcers) or events or settings that exist prior to the occurrence of the problem behavior (i.e., antecedents). Indirect assessments regarding Julian's behavior were then mailed home and completed by legal guardians prior to his appointment. These assessments included the Motivation Assessment Scale (MAS; Durand and Crimmins 1992) and the Questions About Behavior Function Form (QABF; Paclawskyj et al. 2000). The MAS collects information regarding potential function of his problem behaviors through 16 items, Likert-type scale of $0-6(0=$ never, $1=$ almost never, $2=$ seldom, $3=$ half the time, $4=$ usually, $5=$ almost always, $6=$ always). Primary, and possible secondary, functions of the behavior are determined by the MAS by ranking and organizing scores obtained into four potential functions: sensory, escape, attention, and tangible. The QABF measures conditions and events under which behavior is more likely to occur through a 25 -item, Likert-type scale of $0-3(0=$ never, $1=$ 
rarely, $2=$ some, $3=$ often) and includes five categories reflecting behavioral function of attention, escape, non-social, physical, and tangible. The category receiving the highest score suggests the function of targeted problem behavior.

After Julian and his parents arrived at the clinic, a brief functional analysis (FA) was conducted to determine the function, or controlling environmental variables, of his problematic behavior (Northup et al. 1991). This FA involved systematic manipulation of variables that mirror Julian's everyday life which are thought to maintain or cause his behavior. The test conditions were run with Julian and his parents in the examination room and the treatment conditions were run with Julian, his parents, and the therapist. During all ten sessions, each instance of problem behavior was recorded via paper and pencil within 10-s intervals for each session. Then, the rate of problem behavior (physical aggression, self-injurious behavior, and elopement) was calculated by dividing the total number of instances of problem behavior by the number of minutes of the session $(5 \mathrm{~min})$. The percentage of compliance was also calculated by taking the number of correct responses provided by Julian and dividing that number by the total number of instructions delivered by his parent and/or therapist.

Four test conditions that targeted pre-identified functions (per the indirect assessments), each lasting $5 \mathrm{~min}$, were run. The test conditions included control, tangible, demand, and divided-attention conditions (Iwata et al. 1982/1994). A control or play condition was run three times and consisted of access to preferable items and attention from his parent with no demands or tasks placed on the patient. The tangible condition consisted of Julian playing with a high-preference item for $15 \mathrm{~s}$ and then the item was removed; however, the item was returned to him contingent upon problem behavior. During the demand condition, Julian was asked by his parent to complete a task, and if a problem behavior occurred, he was given a 15-s break before being asked to complete the task again. Finally, both parents were present during the divided attention condition which consisted of the parents talking to one another and ignoring Julian, but if problem behaviors occurred attention from both parents was delivered to him.

Additionally, three treatment conditions, each lasting $5 \mathrm{~min}$, were conducted to address the identified functions of behavior (Anderson et al. 1999): demand treatment, tangible treatment, and divided attention treatment conditions. During the demand treatment condition, each demand completed resulted in a visual display of progress towards a break (numbers "1," "2," " 3 " were removed from a strip). A break was provided once three tasks were completed. Given the frequency with which problem behaviors occurred in the initial demand condition, the demand treatment condition was run twice. In the tangible treatment condition, Julian was given access to the high-preference item for $30 \mathrm{~s}$ and when a timer sounded, Julian was prompted to give the item to his parent.
Julian then waited for $30 \mathrm{~s}$ to play with the item again. Each time he gave his parent access to the item, praise and a reinforcer (a single fruit snack) were delivered. Completion of a 30-s period was not contingent upon the absence of problem behavior. During the divided attention treatment condition, Julian was given a container full of novel items he was reported to enjoy while his parents talked. Every $30 \mathrm{~s}$, his parents would deliver praise to Julian for playing with his toys. Completion of a 30 -s period was not contingent upon the absence of problem behavior. Across all treatment conditions, problem behaviors that occurred were ignored.

\section{Results}

During the Functional Assessment Interview with Julian's mother, problem behaviors were identified. The responses from the interview lead clinicians to hypothesize that Julian's problem behaviors were motivated by gaining access to tangible items and by escaping aversive tasks or demands. Table 1 presents the results from the indirect assessments completed by Julian's parents. The MAS results suggested Julian's problem behaviors were most highly motivated by gaining access to tangible items. The results of the QABF suggested that problem behaviors were motived by tangible items and attention (i.e., positive reinforcement). Both the MAS and QABF reflected that Julian's problem behaviors were primarily reinforced through positive reinforcement, i.e., gaining access to preferred items.

As illustrated in Fig. 1, problem behaviors occurred at the highest rate in the demand condition. There were a total of 26 instances of problem behavior in the 5-min session for a rate of 5.2 problem behaviors per minute (rpm). Problem behaviors also occurred in the tangible and divided attention conditions ( 1.05 and $0.8 \mathrm{rpm}$, respectively), although at a lower rate. These behaviors decreased in the demand treatment (for both sessions problem behavior occurred at $0.2 \mathrm{rpm}$ ), tangible treatment, and divided attention treatment conditions

Table 1 Results from MAS, QABF, and FAI completed by the patient's parents regarding patient's problem behaviors (top hypothesized functions of problem behaviors identified through each assessment is in italic)

\begin{tabular}{lll}
\hline $\begin{array}{l}\text { MAS scores } \\
\text { (highest possible score: 6) }\end{array}$ & $\begin{array}{l}\text { QABF scores } \\
\text { (highest possible score: 15) }\end{array}$ & FAI \\
\hline Sensory 3.25 & Attention 10 & Tangible \\
Escape 3.0 & Escape 9 & \\
Attention 2.75 & Non-social 7 \\
Tangible 4.50 & Physical 5 & \\
& Tangible 12 & \\
\hline
\end{tabular}


Fig. 1 Rate of total problem behaviors per minute during conditions across all sessions (demand treatment condition was run twice)

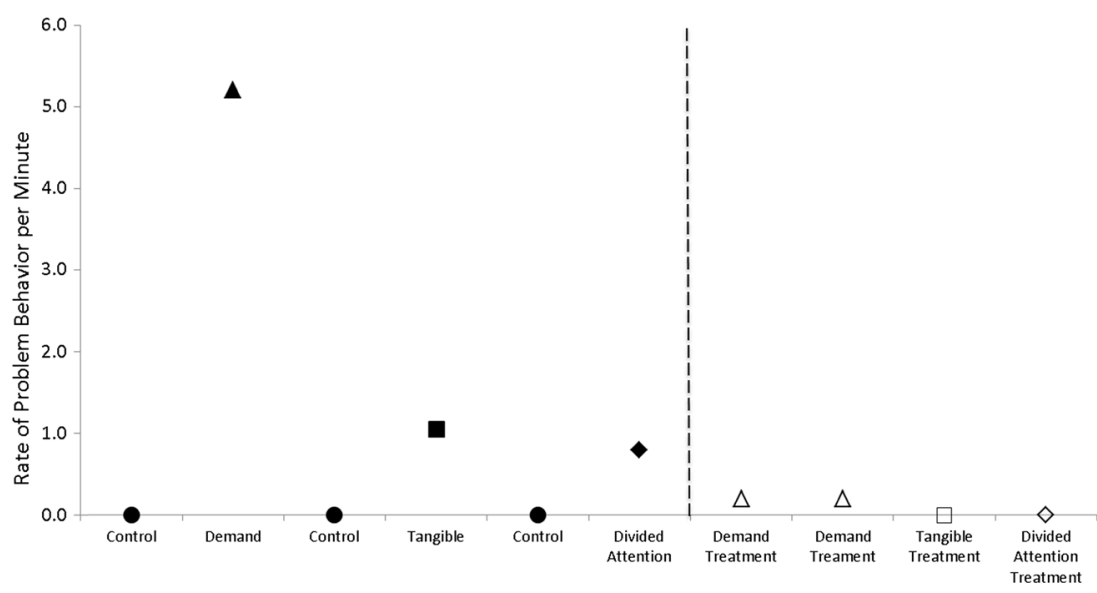

(zero occurrence of problem behavior in these last two conditions). The percentage of compliance was also calculated and found to increase in both demand treatment conditions (79 and $70 \%$ compliance, respectively) when compared to the initial demand condition (14\% compliance; see Fig. 2).

\section{Discussion}

All of the completed assessments yielded similar outcomes; that is, Julian's problem behaviors occurred most often under conditions where behavior produced escape from aversive situations or tasks and to lesser extent when they produced access to tangible and social reinforcement. The FA showed high rates of problem behaviors in the demand, tangible, and divided attention conditions which dramatically decreased in the treatment conditions. Julian's behaviors are consistent with reported behavioral problems seen in individuals with $4 q 21$ microdeletion. The decrease in frequency with which he engaged in problem behavior during the demand condition when compared to the demand treatment conditions was significant. Julian went from engaging in more than five instances of problem behavior per minute in the demand condition to one behavior throughout the entire 5-min session in each of the demand treatment conditions. Furthermore, as decreases in Julian's problem behaviors were observed in the treatment conditions, increases in compliance with instructions were also observed.

Although, limited information is available regarding the behavioral phenotype of this type of genetic syndrome; Julian's problem behaviors, specifically his aggression and SIB, correspond with reports of other individuals diagnosed with 4q21 microdeletion syndrome (Bonnet et al. 2010; Strehle et al. 2012; Tsang et al. 2012). Just as functional analyses have been used to successfully target the function of problem behaviors and to form individualized
Fig. 2 Percentage of compliance with task demands during demand and demand treatment conditions (demand treatment condition was run twice)

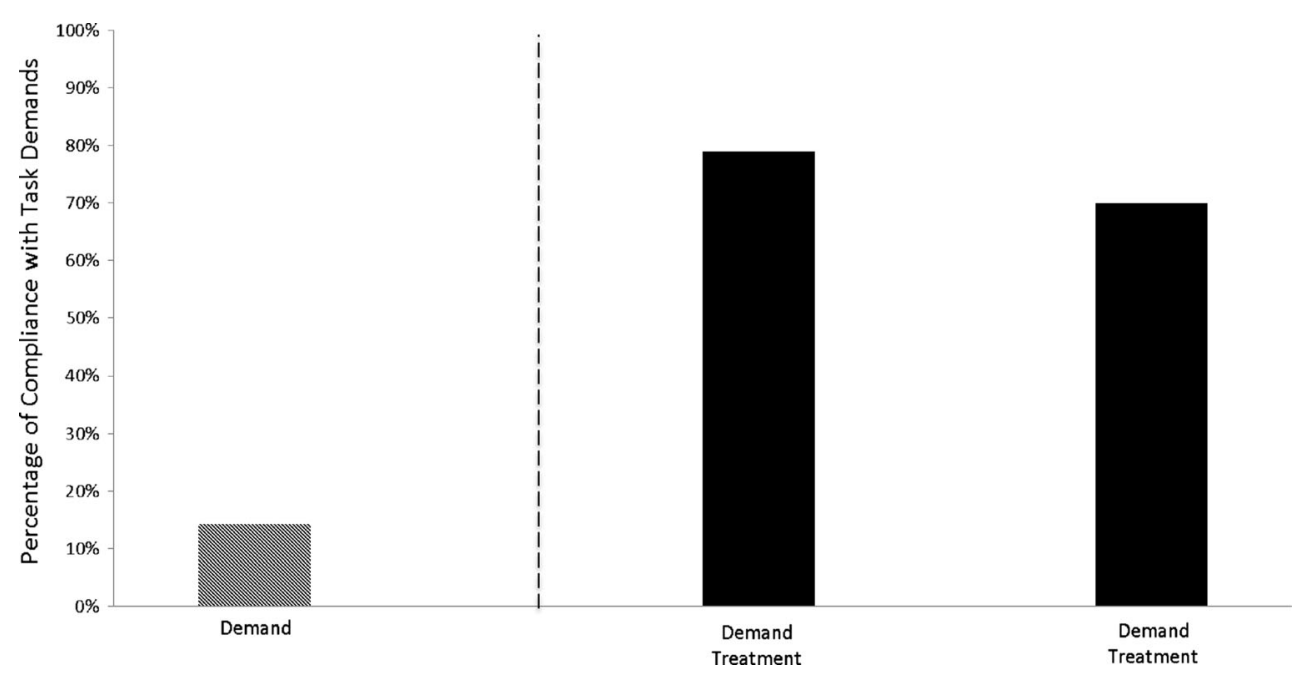


recommendations in conditions associated with intellectual disability (Iwata et al. 1982/1994), functional analysis methodology appears to be a viable option for uncovering the variables maintaining problem behavior associated with this condition as well.

As an aside, prior to our assessment, Julian had been maintained on a steady dose of aripiprazole. Past research has shown decreases in problem behaviors in tangible and demand conditions associated with atypical antipsychotic use, specifically aripiprazole (Danov et al.2012). However, conclusions cannot be made concerning Julian's behavior and the effects of the medication. Nonetheless, future research should consider the interactive effects of medication on behavior function of individuals with this microdeletion or any other syndrome.

Although research has demonstrated that brief functional analyses yield results consistent with longer and extended functional analyses (Tincani, Castrogiavanni,\& Axelrod, 1999), extended analyses in this study would have allowed for running conditions to determine if self-injurious behavior was automatically maintained (i.e., non-socially mediated) versus multiply controlled (i.e., many functions) and for the replication of treatment effects across all interventions applied (i.e., treatment conditions). Unfortunately, given the nature of the appointment, our time with the patient was limited. We were also unable to calculate interobserver agreement as data were collected live during the assessment; however, given that the behavioral phenotype associated with other genetic syndromes have been amenable to behavioral assessment and treatment, we feel this study demonstrates that the behaviors associated with 4q21 microdeletion syndrome may also be influenced by reinforcement contingencies. Thus, future research should be conducted to confirm that behaviors associated with this condition are indeed amenable to socially mediated contingencies of reinforcement. Should future research yield findings consistent with those reported here, additional research should be conducted to determine if behavioral interventions for problem behaviors observed in children with $4 \mathrm{q} 21$ microdeletion syndrome can be successful without the adjunctive use of psychotropic medication.

\section{Compliance with Ethical Standards}

Ethics Approval All procedures followed were in accordance with the ethical standards of the responsible committee on human experimentation (institutional and national) and with the Helsinki Declaration of 1975, as revised in 2000 .

Consent to Participate Informed consent was obtained from legal guardians for the use of these data for purposes of publication.

Conflict of Interest The authors declare that they have no competing interests.

\section{References}

Anderson, C. M., Freeman, K. A., \& Scotti, J. R. (1999). Evaluation of the generalizability (reliability and validity) of analog functional assessment methodology. Behavior Therapy, $30,31-50$.

Barth, A., Bilkei-Gorzo, A., Drews, E., Otte, D. M., Diaz-Lacava, A., Varadarajulu, J., \& Zimmer, A. (2014). Analysis of quantitative trait loci in mice suggests a role of ENOPH1 in stress reactivity. Journal of Neurochemistry, 128, 807-817.

Bhoj, E., Halbach, S., McDonald-McGinn, D., Tan, C., Lande, R., Waggoner, D., \& Zackai, E. (2013). Expanding the spectrum of microdeletion 4q21 syndrome: a partial phenotype with incomplete deletion of the minimal critical region and a new association with cleft palate and Pierre Robin sequence. American Journal of Medical Genetics, 161, 2327-2333.

Bonnet, C., Andrieux, J., Bério-Dexheimer, M., Leheup, B., Boute, O., Manouvrier, S., \& Sanlaville, D. (2010). Microdeletion at chromosome 4q21 defines new emerging syndrome with marked growth restriction, mental retardation and absent or severely delayed speech. Journal of Medical Genetics, 47, 377-384.

Danov, S. E., Tervo, R., Meyers, S., \& Symons, F. J. (2012). Using functional analysis methodology to evaluate effects of an atypical antipsychotic on severe problem behavior. Journal of Mental Health Research in Intellectual Disabilities, 5, 286-308.

Dukes-Rimsky, L., Guzauskas, G. F., Holden, K. R., Griggs, R., Ladd, S., Carmen Montoya, M., \& Srivastava, A. K. (2010). Microdeletion at $4 \mathrm{q} 21.3$ is associated with intellectual disability, dysmorphic facies, hypotonia, and short stature. Journal of Medical Genetics, 155, 2146-2153.

Durand, M. V., \& Crimmins, D. B. (1992). The motivation assessment scale administration guide. Topeka, KS: Monaco \& Associates.

Gudbjartsson, D. F., Walters, G. B., Thorleifsson, G., Stefansson, H., Halldorsson, B. V., Zusmanovich, P., \& Steffanson, K. (2008). Many sequence variants affecting diversity of adult human height. Nature Genetics, 40, 609-615.

Iwata, B. A., Dorsey, M. F., Slifer, K. J., Bauman, K. E., \& Richman, G. S. (1982/1994). Toward a functional analysis of self-injury. Journal of Applied Behavior Analysis, 27, 197-209. (Reprinted from Analysis and Intervention in Developmental Disabilities, 2, 3-20.

Nigro, V., \& Savarese, M. (2014). Genetic basis of limb-girdle muscular dystrophies: the 2014 update. Acta Myologica, 33, 1-12.

Northup, J., Wacker, D., Sasso, G., Steege, M., Cigrand, K., Cook, J., \& DeRaad, A. (1991). A brief functional analysis of aggressive and alternative behavior in an out clinic setting. Journal of Applied Behavior Analysis, 24, 509-522.

O’Neill, R. E., Homer, R. H., Albin, R. W., Sprague, J. R., Storey, K., \& Newton, J. S. (1997). Functional assessment and program development for problem behavior. Pacific Grove, CA: Brooks/Cole Publishing.

Paclawskyj, T. R., Matson, J. L., Rush, K. S., Smalls, Y., \& Vollmer, T. R. (2000). Questions About Behavioral Function (QABF): a behavioral checklist for functional assessment of aberrant behavior. Research in Developmental Disabilities, 21, 223-229.

Roane, H., Bouxsein, K., \& Fulton, C. (2012). Assessment and treatment of self-injurious behavior associated with Donnai-Barrow syndrome. Journal of Developmental and Physical Disabilities, 24, 327-335.

Sloneem, J., Oliver, C., Udwin, O., \& Woodcock, K. A. (2011). Prevalence, phenomenology, aetiology and predictors of challenging behaviour in Smith-Magenis syndrome. Journal of Intellectual Disability Research, 55, 138-151.

Strehle, E. M., Yu, L., Rosenfeld, J. A., Donkervoort, S., Zhou, Y., Chen, T. J., \& Huang, T. (2012). Genotype-phenotype analysis of $4 \mathrm{q}$ 
deletion syndrome: proposal of a critical region. American Journal of Medical Genetics Part A, 158A, 2139-2151.

Tincani, M. J., Castrogiavanni, A., \& Axelrod, S. (1999). A comparison of the effectiveness of brief versus traditional functional analyses. Research in Developmental Disabilities, 20, 327-338.
Tsang, E., Rupps, R., McGillivray, B., Eydoux, P., Marra, M., Arbour, L., \& Zahir, F. R. (2012). Life-history chronical for a patient with the recently described chromosome $4 \mathrm{q} 21$ microdeletion syndrome. American Journal of Medical Genetics Part A, 158A, 2606-2609. 